\title{
Erratum to: A multicenter, open-label, dose-ranging study to exploratively evaluate the efficacy, safety, and dose-response of tolvaptan in patients with decompensated liver cirrhosis
}

\author{
Kiwamu Okita · Isao Sakaida • Mitsuru Okada • Akira Kaneko • \\ Kazuaki Chayama - Michio Kato $\cdot$ Michio Sata - Harumasa Yoshihara • \\ Noriyuki Ono $\cdot$ Yoshikazu Murawaki
}

Published online: 15 May 2010

(C) Springer 2010

\section{Erratum to: J Gastroenterol \\ DOI 10.1007/s00535-010-0240-6}

The name of the eighth author should be given as Harumasa Yoshihara, not Naomasa Yoshihara.

The author's affiliation and e-mail address also appeared incorrectly. The correct affiliation and e-mail address are as follows:

The online version of the original article can be found under doi: 10.1007/s00535-010-0240-6.

\section{K. Okita $(\bowtie)$}

Social Insurance Alliance Shimonoseki Kohsei Hospital,

3-3-8 Kamishinchi-cho, Shimonoseki,

Yamaguchi 750-0061, Japan

e-mail: k.okita@kousei-h.jp

\section{Sakaida}

Department of Gastroenterology and Hepatology,

Yamaguchi University Graduate School of Medicine,

Minami Kogushi 1-1-1, Ube, Yamaguchi 755-8505, Japan

e-mail: sakaida@yamaguchi-u.ac.jp

\section{Okada}

Otsuka Pharmaceutical Co., Ltd., Osaka, Japan

e-mail: okadamit@otsuka.jp

\author{
A. Kaneko \\ Department of Internal Medicine, \\ NTT West Osaka Hospital, Osaka, Japan \\ e-mail: a.kaneko@mhc.west.ntt.co.jp

\section{K. Chayama} \\ Division of Frontier Medical Science, \\ Department of Molecular Science, \\ Programs for Biomedical Research, Graduate School \\ of Biomedical Science, Hiroshima University, Hiroshima, Japan \\ e-mail: chayama@hiroshima-u.ac.jp
}

\author{
H. Yoshihara \\ Department of Internal Medicine, \\ Osaka Rosai Hospital, Osaka, Japan \\ e-mail: hyoshihara@orh.go.jp
}

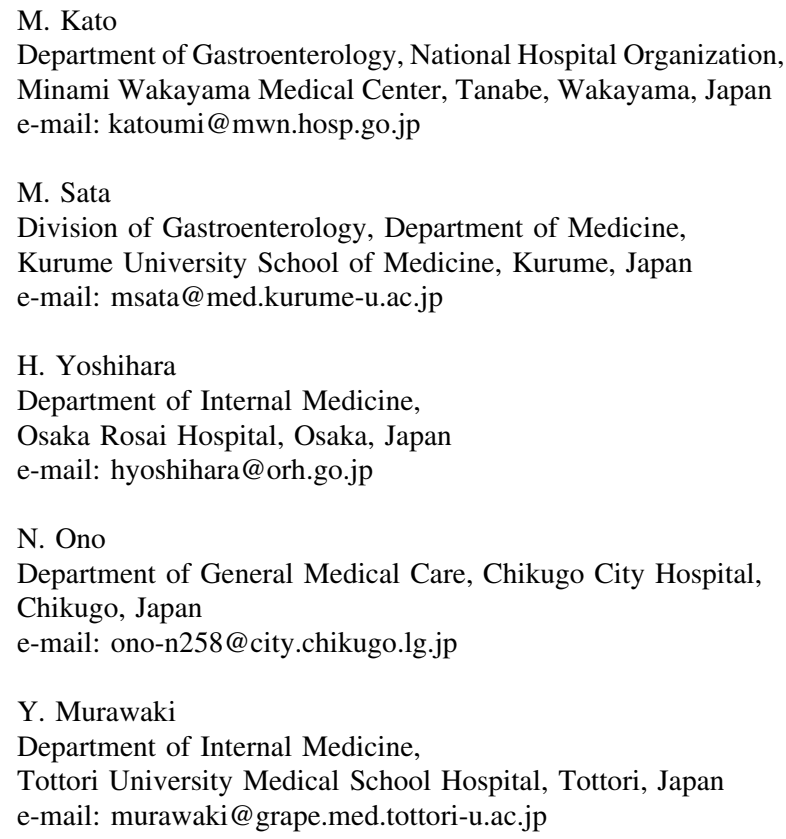
Minami Wakayama Medical Center, Tanabe, Wakayama, Japan e-mail:katoumi@mwn.hosp.go.jp

M. Sata

Division of Gastroenterology, Department of Medicine, Kurume University School of Medicine, Kurume, Japan e-mail: msata@med.kurume-u.ac.jp

H. Yoshihara

Department of Internal Medicine,

Osaka Rosai Hospital, Osaka, Japan

e-mail: hyoshihara@orh.go.jp

N. Ono

Department of General Medical Care, Chikugo City Hospital,

Chikugo, Japan

e-mail: ono-n258@ city.chikugo.lg.jp

Y. Murawaki

Department of Internal Medicine,

Tottori University Medical School Hospital, Tottori, Japan

e-mail: murawaki@grape.med.tottori-u.ac.jp 